\title{
Public Key Compression and Modulus Switching for Fully Homomorphic Encryption over the Integers
}

\author{
Jean-Sébastien Coron ${ }^{1}$, David Naccache ${ }^{2}$, and Mehdi Tibouchi ${ }^{3}$ \\ 1 Université du Luxembourg \\ jean-sebastien.coron@uni.lu \\ 2 École normale supérieure \\ david.naccache@ens.fr \\ 3 NTT Information Sharing Platform Laboratories \\ tibouchi.mehdi@lab.ntt.co.jp
}

\begin{abstract}
We describe a compression technique that reduces the public key size of van Dijk, Gentry, Halevi and Vaikuntanathan's (DGHV) fully homomorphic scheme over the integers from $\tilde{\mathcal{O}}\left(\lambda^{7}\right)$ to $\tilde{\mathcal{O}}\left(\lambda^{5}\right)$. Our variant remains semantically secure, but in the random oracle model. We obtain an implementation of the full scheme with a $10.1 \mathrm{MB}$ public key instead of $802 \mathrm{MB}$ using similar parameters as in [7. Additionally we show how to extend the quadratic encryption technique of [7] to higher degrees, to obtain a shorter public-key for the basic scheme.

This paper also describes a new modulus switching technique for the DGHV scheme that enables to use the new FHE framework without bootstrapping from Brakerski, Gentry and Vaikuntanathan with the DGHV scheme. Finally we describe an improved attack against the Approximate GCD Problem on which the DGHV scheme is based, with complexity $\tilde{\mathcal{O}}\left(2^{\rho}\right)$ instead of $\tilde{\mathcal{O}}\left(2^{3 \rho / 2}\right)$.
\end{abstract}

\section{Introduction}

Fully Homomorphic Encryption. An encryption scheme is said to be fully homomorphic when it is possible to perform implicit plaintext additions and multiplications while manipulating only ciphertexts.

The first construction of a fully homomorphic scheme was described by Gentry in 9]. Gentry first obtained a "somewhat homomorphic" scheme, supporting only a limited number of ciphertext multiplications due to the fact that ciphertext contain a certain amount of "noise" which increases with every multiplication, and that decryption fails when noise size passes a certain bound. As a result, in the somewhat homomorphic scheme, the functions that can be homomorphically evaluated on ciphertexts are polynomials of small, bounded degree. The second step in Gentry's framework consists in "squashing" the decryption procedure so that it can be expressed as a low degree polynomial in the bits of the ciphertext and the secret key. Then, Gentry's key idea, called "bootstrapping", is to evaluate this decryption polynomial not on the ciphertext bits and the secret-key 
bits (which would yield the plaintext), but homomorphically on the encryption of those bits, which gives another ciphertext of the same plaintext. If the degree of the decryption polynomial is small enough, the noise in the new ciphertext can become smaller than it was the original ciphertext, so that this new ciphertext can be used again in a subsequent homomorphic operation (either addition or multiplication). Using this "ciphertext refresh" procedure the number of permissible homomorphic operations becomes unlimited and one obtains a fully homomorphic encryption scheme. To date, three different fully homomorphic schemes are known:

1. Gentry's original scheme [9], based on ideal lattices. Gentry and Halevi described in [10] the first implementation of Gentry's scheme, using many clever optimizations, including some suggested in a previous work by Smart and Vercauteren [14]. For their most secure setting (claiming 72 bit security) the authors report a public key size of $2.3 \mathrm{~GB}$ and a ciphertext refresh procedure taking 30 minutes on a high-end workstation.

2. van Dijk, Gentry, Halevi and Vaikuntanathan's (DGHV) scheme over the integers $[8$. This scheme is conceptually simpler than Gentry's scheme, because it operates on integers instead of ideal lattices. Recently it was shown 7. how to reduce the public key size by storing only a small subset of the original public key and generating the full public key on the fly by combining the elements in the small subset multiplicatively. Using some of the optimizations from [10, the authors of 7 report similar performances: a 802 MB public key and a ciphertext refresh in 14 minutes.

3. Brakerski and Vaikuntanathan's scheme based on the Learning with Errors (LWE) and Ring Learning with Errors (RLWE) problems [2]3. The authors introduce a new dimension reduction technique and a new modulus switching technique to shorten the ciphertext and reduce the decryption complexity. A partial implementation is described in [11, without the fully homomorphic capability.

Recently Brakerski, Gentry and Vaikuntanathan introduced a remarkable new FHE framework, in which the noise ceiling increases only linearly with the multiplicative level instead of exponentially [4; this implies that bootstrapping is no longer necessary to achieve fully homomorphic encryption. This new framework has the potential to significantly improve the practical FHE performance. The new framework is based on Brakerski and Vaikuntanathan's scheme [2], and more specifically on their new modulus switching technique, which efficiently transforms a ciphertext encrypted under a certain modulus $p$ into a ciphertext under a different modulus $p^{\prime}$ but with reduced noise.

Public Key Compression. The first of our contributions is a technique to reduce the public key size of DGHV-like schemes [8] by several orders of magnitude. In the DGHV scheme the public key is a set of integers of the form:

$$
x_{i}=q_{i} \cdot p+r_{i}
$$


where $p$ is the secret-key of $\eta$ bits, $q_{i}$ is a large random integer of $\gamma-\eta$ bits, and $r_{i}$ is a small random integer of $\rho$ bits. The scheme's semantic security is based on the Approximate GCD Problem: given a polynomial number of $x_{i}$ 's, recover the secret $p$. To avoid lattice attacks, the bit-size $\gamma$ of the $x_{i}$ 's must be very large: [7. takes $\gamma \simeq 2 \cdot 10^{7}$ for $\eta=2652$ and $\rho=39$, and the full public key claims a $802 \mathrm{MB}$ storage.

Our technique proceeds as follows. First generate the secret-key $p$. Then, use a pseudo-random number generator $f$ with public random seed se to generate a set of $\gamma$-bit integers $\chi_{i}$ (i.e. the $\chi_{i}$ 's are of the same bit-size as the $x_{i}$ 's). Finally, compute small corrections $\delta_{i}$ to the $\chi_{i}$ 's such that $x_{i}=\chi_{i}-\delta_{i}$ is small modulo $p$, and store only the small corrections $\delta_{i}$ in the public key, instead of the full $x_{i}$ 's. Knowing the PRNG seed se and the $\delta_{i}$ 's is sufficient to recover the $x_{i}$ 's.

Therefore instead of storing a set of large $\gamma$-bit integers we only have to store a set of much smaller $\eta$-bit integers, where $\eta$ is the bit size of $p$. The new technique is fully compatible with the DGHV variant described in 77; with the previous set of parameters from [7] one obtains a public key size of 4.6 MB for the full implementation, instead of the $802 \mathrm{MB}$ required in [7]! The technique can be seen as generating the $\gamma-\eta$ most significant bits of the $x_{i}$ 's with a pseudo-random number generator, and then using the secret key $p$ to fix the $\eta$ remaining bits so that $x_{i} \bmod p$ is small. While different, this is somewhat reminiscent of Lenstra's technique 12 for generating an RSA modulus with a predetermined portion.

Under our variant, the encryption scheme can still be proved semantically secure under the Approximate GCD assumption, albeit in the random oracle model. This holds for both the original DGHV scheme form [8] and the variant described in [7] in which the public key elements are first combined multiplicatively to generate the full public key. Unlike 788 , we need the random oracle model in order to apply the leftover hash lemma in our variant, because the seed of the PRNG is known to the attacker (as part of the public key).

We report the result of an implementation of the new variant with the fully homomorphic capability. As in [7] we use the variant with noise-free $x_{0}=q_{0} \cdot p$. We also update the parameters from [7] to take into the account the improved attack from Chen and Nguyen against the Approximate GCD problem [5]. We obtain a level of efficiency very similar to [7] but with a 10.1 MB public key instead of a $802 \mathrm{MB}$ one. The source code of this implementation is publicly available [17.

Extension to Higher Degrees. Various techniques have been proposed in [7] to reduce the public key size and increase the efficiency of the DGHV scheme, the most important of which is to use a quadratic form instead of a linear form for masking the message when computing a ciphertext. The authors show that the scheme remains semantically secure; the key ingredient is to prove that a certain family of quadratic hash functions is close enough to being pairwise independent, so that the leftover hash lemma can still be applied. The main benefit is a significant reduction in public key size, from $\tau=\tilde{\mathcal{O}}\left(\lambda^{3}\right)$ elements $x_{i}$ down to $2 \beta=\tilde{\mathcal{O}}\left(\lambda^{1.5}\right)$ elements $x_{i, b}$. In this paper we prove that the natural 
extension of this quadratic encryption technique to to cubic forms, and more generally forms of arbitrary fixed degree $d$, remains secure, making it possible to further reduce the public key size.

Modulus Switching and Leveled DGHV Scheme. As a third contribution, we show how to adapt Brakerski, Gentry and Vaikuntanathan's (BGV) new FHE framework 44 to the DGHV scheme over the integers. Under the BGV framework the noise ceiling increases only linearly with multiplicative depth, instead of exponentially. This enables to get a FHE scheme without the costly bootstrapping procedure.

More precisely the new BGV framework is described in [4] with Brakerski and Vaikuntanathan's scheme [2], and the key technical tool is the modulus-switching technique of 2] that transforms a ciphertext $c$ modulo $p$ into a ciphertext $\boldsymbol{c}^{\prime}$ modulo $p^{\prime}$ simply by scaling by $p^{\prime} / p$ and rounding appropriately. This allows to reduce the ciphertext noise by a factor close to $p^{\prime} / p$ without knowing the secret-key and without bootstrapping. However the modulus switching technique cannot directly apply to DGHV since in DGHV the moduli $p$ and $p^{\prime}$ are secret. In this paper we explain how this modulus-switching technique can be adapted to DGHV, so as to apply the new BGV framework. We show that the resulting FHE scheme remains semantically secure, albeit under a stronger assumption. We also describe an implementation, showing that the new BGV framework can be applied in practice.

Improved Attack against the Approximate-GCD problem. Finally we consider the security of the Approximate GCD Problem without noise-free $x_{0}=$ $q_{0} \cdot p$. In our leveled DGHV variant under the BGV framework the size of the secret $p$ can become much smaller than in the original Gentry framework $(\eta \simeq 180$ bits for the lowest $p$ in the ladder, instead of $\eta=2652$ bits in [7]). This implies that the noise-free variant $x_{0}=q_{0} \cdot p$ cannot be used, since otherwise the prime factor $p$ could easily be extracted using the Elliptic Curve Method for integer factorization [13]. Therefore one must consider the security of the Approximate GCD Problem without noise-free $x_{0}$. The recent attack by Chen and Nguyen [5] against the Approximate GCD Problem with noise-free $x_{0}$ has complexity $\tilde{\mathcal{O}}\left(2^{\rho / 2}\right)$, instead of the $\tilde{\mathcal{O}}\left(2^{\rho}\right)$ naive attack; as noted by the authors, this immediately yields an $\tilde{\mathcal{O}}\left(2^{3 \rho / 2}\right)$ attack against the Approximate GCD Problem without noise-free $x_{0}$, instead of $\tilde{\mathcal{O}}\left(2^{2 \rho}\right)$ for the naive attack. In this paper we exhibit an improved attack with complexity $\tilde{\mathcal{O}}\left(2^{\rho}\right)$. We also describe an implementation showing that this new attack is indeed an improvement in practice.

\section{The DGHV Scheme over the Integers}

We first recall the somewhat homomorphic encryption scheme described by van Dijk, Gentry, Halevi and Vaikuntanathan (DGHV) in $[\underline{8}$. For a real number $x$, we denote by $\lceil x\rceil,\lfloor x\rfloor$ and $\lceil x\rfloor$ the rounding of $x$ up, down, or to the nearest integer. For integers $z, p$ we denote the reduction of $z$ modulo $p$ by $[z]_{p}$ with $-p / 2<[z]_{p} \leq p / 2$, and by $\langle z\rangle_{p}$ with $0 \leq\langle z\rangle_{p}<p$. Given the security parameter $\lambda$, the following parameters are used: 
- $\gamma$ is the bit-length of the $x_{i}$ 's,

- $\eta$ is the bit-length of the secret key $p$,

- $\rho$ is the bit-length of the noise $r_{i}$,

- $\tau$ is the number of $x_{i}$ 's in the public key,

- $\rho^{\prime}$ is a secondary noise parameter used for encryption.

For a specific $\eta$-bit odd integer $p$, we use the following distribution over $\gamma$-bit integers:

$\mathcal{D}_{\gamma, \rho}(p)=\left\{\right.$ Choose $q \leftarrow \mathbb{Z} \cap\left[0,2^{\gamma} / p\right), r \leftarrow \mathbb{Z} \cap\left(-2^{\rho}, 2^{\rho}\right):$ Output $\left.x=q \cdot p+r\right\}$

DGHV. KeyGen $\left(1^{\lambda}\right)$. Generate a random prime integer $p$ of size $\eta$ bits. For $0 \leq$ $i \leq \tau$ sample $x_{i} \leftarrow \mathcal{D}_{\gamma, \rho}(p)$. Relabel the $x_{i}$ 's so that $x_{0}$ is the largest. Restart unless $x_{0}$ is odd and $\left[x_{0}\right]_{p}$ is even. Let $p k=\left(x_{0}, x_{1}, \ldots x_{\tau}\right)$ and $s k=p$.

DGHV. Encrypt $(p k, m \in\{0,1\})$. Choose a random subset $S \subseteq\{1,2, \ldots, \tau\}$ and a random integer $r$ in $\left(-2^{\rho^{\prime}}, 2^{\rho^{\prime}}\right)$, and output the ciphertext:

$$
c=\left[m+2 r+2 \sum_{i \in S} x_{i}\right]_{x_{0}}
$$

DGHV.Evaluate $\left(p k, C, c_{1}, \ldots, c_{t}\right)$ : given the circuit $C$ with $t$ input bits, and $t$ ciphertexts $c_{i}$, apply the addition and multiplication gates of $C$ to the ciphertexts, performing all the additions and multiplications over the integers, and return the resulting integer.

DGHV. Decrypt $(s k, c)$. Output $m \leftarrow[c]_{p} \bmod 2$.

This completes the description of the scheme. As shown in 8 this scheme is somewhat homomorphic, i.e. a limited number of homomorphic operations can be performed on ciphertexts. More precisely given two ciphertexts $c=q \cdot p+$ $2 r+m$ and $c^{\prime}=q^{\prime} \cdot p+2 r^{\prime}+m^{\prime}$ where $r$ and $r^{\prime}$ are $\rho^{\prime}$-bit integers, the ciphertext $c+c^{\prime}$ is an encryption of $m+m^{\prime} \bmod 2$ with $\left(\rho^{\prime}+1\right)$-bit noise and the ciphertext $c \cdot c^{\prime}$ is an encryption of $m \cdot m^{\prime}$ with noise $\simeq 2 \rho^{\prime}$. Since the ciphertext noise must remain smaller than $p$ for correct decryption, the scheme allows roughly $\eta / \rho^{\prime}$ multiplications on ciphertexts. As shown in 8 the scheme is semantically secure under the Approximate GCD assumption.

Definition 1 (Approximate GCD). The ( $\rho, \eta, \gamma)$-Approximate GCD Problem is: For a random $\eta$-bit odd integer $p$, given polynomially many samples from $\mathcal{D}_{\gamma, \rho}(p)$, output $p$.

\section{The New DGHV Public Key Compression Technique}

We describe our technique using the variant with noise free $x_{0}=q_{0} \cdot p$, as suggested in 8 and implemented in 7. We only describe the basic scheme; we refer to the full version of this paper [6] for a complete description of the fully homomorphic scheme. 


\subsection{Description}

KeyGen $\left(1^{\lambda}\right)$. Generate a random prime integer $p$ of size $\eta$ bits. Pick a random odd integer $q_{0} \in\left[0,2^{\gamma} / p\right)$ and let $x_{0}=q_{0} \cdot p$. Initialize a pseudo-random number generator $f$ with a random seed se. Use $f(\mathrm{se})$ to generate a set of integers $\chi_{i} \in$ $\left[0,2^{\gamma}\right)$ for $1 \leq i \leq \tau$. For all $1 \leq i \leq \tau$ compute:

$$
\delta_{i}=\left\langle\chi_{i}\right\rangle_{p}+\xi_{i} \cdot p-r_{i}
$$

where $r_{i} \leftarrow \mathbb{Z} \cap\left(-2^{\rho}, 2^{\rho}\right)$ and $\xi_{i} \leftarrow \mathbb{Z} \cap\left[0,2^{\lambda+\eta} / p\right)$. For all $1 \leq i \leq \tau$ compute:

$$
x_{i}=\chi_{i}-\delta_{i}
$$

Let $p k=\left(\mathrm{se}, x_{0}, \delta_{1}, \ldots, \delta_{\tau}\right)$ and $s k=p$.

$\operatorname{Encrypt}(p k, m \in\{0,1\})$ : use $f($ se $)$ to recover the integers $\chi_{i}$ and let $x_{i}=\chi_{i}-\delta_{i}$ for all $1 \leq i \leq \tau$. Choose a random integer vector $\boldsymbol{b}=\left(b_{i}\right)_{1 \leq i \leq \tau} \in\left[0,2^{\alpha}\right)^{\tau}$ and a random integer $r$ in $\left(-2^{\rho^{\prime}}, 2^{\rho^{\prime}}\right)$. Output the ciphertext:

$$
c=m+2 r+2 \sum_{i=1}^{\tau} b_{i} \cdot x_{i} \quad \bmod x_{0}
$$

Evaluate $\left(p k, C, c_{1}, \ldots, c_{t}\right)$ and $\operatorname{Decrypt}(s k, c)$ : same as in the original DGHV scheme, except that ciphertexts are reduced modulo $x_{0}$.

This completes the description of our variant. We have the following constraints on the scheme parameters:

- $\rho=\omega(\log \lambda)$ to avoid brute force attack on the noise,

- $\eta \geq \rho \cdot \Theta\left(\lambda \log ^{2} \lambda\right)$ in order to support homomorphic operations for evaluating the "squashed decryption circuit" (see [8]),

- $\gamma=\omega\left(\eta^{2} \cdot \log \lambda\right)$ in order to thwart lattice-based attacks against the Approximate GCD problem (see [78]),

- $\alpha \cdot \tau \geq \gamma+\omega(\log \lambda)$ in order to apply the left-over hash lemma (see [7/8]).

- $\eta \geq \rho+\alpha+2+\log _{2} \tau$ for correct decryption of a ciphertext,

- $\rho^{\prime}=\alpha+\rho+\omega(\log \lambda)$ for the secondary noise parameter.

To satisfy the above constraints one can take $\rho=\lambda, \eta=\tilde{\mathcal{O}}\left(\lambda^{2}\right), \gamma=\tilde{\mathcal{O}}\left(\lambda^{5}\right)$, $\alpha=\tilde{\mathcal{O}}\left(\lambda^{2}\right), \tau=\tilde{\mathcal{O}}\left(\lambda^{3}\right)$ and $\rho^{\prime}=\tilde{\mathcal{O}}\left(\lambda^{2}\right)$. The main difference with the original DGHV scheme is that instead of storing the large $x_{i}$ 's in the public key we only store the much smaller $\delta_{i}$ 's. The new public key for the somewhat homomorphic scheme has size $\gamma+\tau \cdot(\eta+\lambda)=\tilde{\mathcal{O}}\left(\lambda^{5}\right)$ instead of $(\tau+1) \cdot \gamma=\tilde{\mathcal{O}}\left(\lambda^{8}\right)$.

Remark 1. We can also compress $x_{0}$ by letting $x_{0}=\chi_{0}-\delta_{0}$ and storing only $\delta_{0}=\left\langle\chi_{0}\right\rangle_{p}+\xi_{0} \cdot p$ in the public-key. 
Remark 2. In the description above we add a random multiple of $p$ to $\left\langle\chi_{i}\right\rangle_{p}$ in the $\delta_{i}$ 's. This is done to obtain a proof of semantic security in the random oracle model (see below). However the scheme seems heuristically secure without adding the random multiple.

Remark 3. For encryption the integers $x_{i}$ need not be stored in memory as they can be generated on the fly when computing the subset sum.

\subsection{Semantic Security}

Theorem 1. The previous encryption scheme is semantically secure under the Approximate GCD assumption with noise-free $x_{0}=q_{0} \cdot p$, in the random oracle model.

The proof is almost the same as in [8]. Given a random oracle $H:\{0,1\}^{*} \rightarrow$ $\mathbb{Z} \cap\left[0,2^{\gamma}\right)$, we assume that the pseudo-random number generation of the $\chi_{i}$ 's is defined as $\chi_{i}=H($ se $\| i)$ for all $1 \leq i \leq \tau$ and we show that the integers $x_{i}$ 's generated in (2) have a distribution statistically close to their distribution in the original DGHV scheme. We refer to the full version of the paper [6] for the proof.

\section{Extension of DGHV Encryption to Higher Degrees}

Various techniques have recently been proposed in [7] to reduce the public key size and increase the efficiency of the DGHV scheme, the most important of which is to use a quadratic form instead of a linear form for masking the message when computing a ciphertext. More precisely, one computes:

$$
c^{*}=m+2 r+2 \sum_{1 \leq i, j \leq \beta} b_{i j} \cdot x_{i, 0} \cdot x_{j, 1} \bmod x_{0}
$$

which is quadratic in the public key elements $x_{i, b}$ instead of linear as in equation (1); here the variant with noise-free $x_{0}=q_{0} \cdot p$ is used. The main benefit is a significant decrease in the public key size, from $\tau=\tilde{\mathcal{O}}\left(\lambda^{3}\right)$ elements $x_{i}$ down to $2 \beta=\tilde{\mathcal{O}}\left(\lambda^{1.5}\right)$ elements $x_{i, b}$. Namely the constraint to apply the left-over hash lemma becomes $\alpha \cdot \beta^{2} \geq \gamma+\omega(\log \lambda)$, so by taking $\alpha=\tilde{\mathcal{O}}\left(\lambda^{2}\right)$ one can take $\beta=\tilde{\mathcal{O}}\left(\lambda^{1.5}\right)$. Combined with our compression technique the public-key size of the somewhat homomorphic scheme becomes $(2 \beta+1) \cdot(\eta+\lambda)=\tilde{\mathcal{O}}\left(\lambda^{3.5}\right)$.

To prove that the scheme remains secure under this modified encryption procedure, the key point in [7] was to prove that the following family of functions $h:\left\{0, \ldots, 2^{\alpha-1}\right\}^{\beta^{2}} \rightarrow \mathbb{Z}_{q_{0}}$ :

$$
h(\boldsymbol{b})=\sum_{1 \leq i_{1}, i_{2} \leq \beta} b_{i_{1} i_{2}} q_{i_{1}}^{(1)} q_{i_{2}}^{(2)} \quad \bmod q_{0} \quad\left(q_{i}^{(j)} \in \mathbb{Z}_{q_{0}}\right)
$$

is close enough to being a pairwise independent (i.e. universal) hash function family (under suitable parameter choices), which in turn makes it possible to apply a variant of the leftover hash lemma. 
In this section we show that it is possible to obtain further efficiency gains by using cubic forms instead, or more generally forms of higher degree $d$, if we can prove an analogue of the previous result for the family $\mathcal{H}_{d}$ of hash functions $h:\left\{0, \ldots, 2^{\alpha-1}\right\}^{\beta^{d}} \rightarrow \mathbb{Z}_{q}$ of the form:

$$
h(\boldsymbol{b})=\sum_{1 \leq i_{1}, \ldots, i_{d} \leq \beta} b_{i_{1}, \ldots, i_{d}} q_{i_{1}}^{(1)} \cdots q_{i_{d}}^{(d)} \quad \bmod q \quad\left(q_{i}^{(j)} \in \mathbb{Z}_{q}\right)
$$

Such a result also leads to the construction of extractors with relatively short seeds, which is an interesting fact in its own right.

We show that this hash function family is indeed close to being pairwise independent for suitable parameters. As in [7, we can prove this in the simpler case when $q=q_{0}$ is prime; the result then follows for all $q_{0}$ without small prime factors. The main result is as follows (we refer to [7] for the definition of $\varepsilon$ pairwise independence). We provide the proof in the full version of this paper [6].

Theorem 2. For an odd prime $q$, the hash function family $\mathcal{H}_{d}$ is $\varepsilon$-pairwise independent, with:

$$
\varepsilon=\frac{(d-1)(d-2)}{\sqrt{q}}+\frac{5 d^{13 / 3}}{q}+\frac{(d-1) \cdot(2 \beta)^{d}}{2^{\left.\alpha \beta^{d-1}(\beta-2-2 / \alpha)\right)}}
$$

Using the variant of the leftover hash lemma from [7, this proves the semantic security of the scheme for any encryption degree $d \geq 2$, with the condition $\alpha \cdot \beta^{d} \geq \gamma+\omega(\log \lambda)$. The constraint for correct decryption becomes $\eta \geq \rho$. $d+\alpha+2+d \cdot \log _{2} \beta$, and $\rho^{\prime}=\rho \cdot d+\alpha+\omega(\log \lambda)$ for the secondary noise parameter. The public-key size for the somewhat homomorphic scheme becomes $(d \cdot \beta+1) \cdot(\eta+\lambda)$. In particular by taking $\beta=3$ and $d=\mathcal{O}(\log \lambda)$, we get a public-key size in $\tilde{\mathcal{O}}\left(\lambda^{2}\right)$ for the somewhat homomorphic scheme.

\section{Adaptation of the BGV Framework to the DGHV Scheme}

\subsection{The BGV Framework for Leveled FHE}

In this section we first recall the new framework from Brakerski, Gentry and Vaikuntanathan (BGV) [4 for leveled fully homomorphic encryption. Under the BGV framework the noise ceiling increases only linearly with the multiplicative depth, instead of increasing exponentially. This implies that bootstrapping is no longer necessary to achieve fully homomorphic encryption. The new framework is based on the Brakerski and Vaikuntanathan RLWE scheme 23]. The key technical tool is the modulus-switching technique from [2 that transforms a ciphertext $\boldsymbol{c}$ modulo $p$ into a ciphertext $\boldsymbol{c}^{\prime}$ modulo $p^{\prime}$ simply by scaling by $p^{\prime} / p$ and rounding appropriately; the noise is also reduced by a factor $p^{\prime} / p$.

In the original Gentry framework [9], the multiplication of two mod- $p$ ciphertexts with noise size $\rho$ gives a ciphertext with noise size $\simeq 2 \rho$; after a second 
multiplication level the noise becomes $\simeq 4 \rho$, then $\simeq 8 \rho$ and so on; the noise size grows exponentially with the number of multiplication levels. The modulus $p$ is a ceiling for correct decryption; therefore if the bit-size of $p$ is $k \cdot \rho$, the noise ceiling is reached after only $\log _{2} k$ levels of multiplication. Fully homomorphic encryption is achieved via bootstrapping, i.e. homomorphically evaluating the decryption polynomial to obtain a refreshed ciphertext.

The breakthrough idea in the BGV framework [4 is to apply the modulusswitching technique after every multiplication level, using a ladder of gradually decreasing moduli $p_{i}$. Start with two mod- $p_{1}$ ciphertexts with noise $\rho$; as previously after multiplication one gets a mod- $p_{1}$ ciphertext with noise $2 \rho$. Now switch to a new modulus $p_{2}$ such that $p_{2} / p_{1} \simeq 2^{-\rho}$; after the switching one gets a mod- $p_{2}$ ciphertext with noise back to $2 \rho-\rho=\rho$ again; one can continue by multiplying two mod- $p_{2}$ ciphertexts, obtain a $2 \rho$-noise mod- $p_{2}$ ciphertext and switch back to a $\rho$-noise mod- $p_{3}$ ciphertext, and so on. With a ladder of $k$ moduli $p_{i}$ of decreasing size $(k+1) \cdot \rho, \ldots, 3 \rho, 2 \rho$ one can therefore perform $k$ levels of multiplication instead of just $\log _{2} k$. In other words the (largest) modulus size $(k+1) \cdot \rho$ grows only linearly with the multiplicative depth; this is an exponential improvement.

As explained in 4, bootstrapping is no longer strictly necessary to achieve fully homomorphic encryption: namely one can always assume a polynomial upper-bound on the number $L$ of multiplicative levels of the circuit to be evaluated homomorphically. However, bootstrapping is still an interesting operation as a bootstrapped scheme can perform homomorphic evaluations indefinitely without needing to specify at setup time a bound on the multiplicative depth. As shown in [4] bootstrapping becomes also more efficient asymptotically in the BGV framework.

\subsection{Modulus-Switching for DGHV}

The modulus-switching technique recalled in the previous section is a very lightweight procedure to reduce the ciphertext noise by a factor roughly $p / p^{\prime}$ without knowing the secret-key and without bootstrapping. However we cannot apply this technique directly to DGHV since in DGHV the moduli $p$ and $p^{\prime}$ must remain secret.

We now describe a technique for switching moduli in DGHV. We proceed in two steps. Given as input a DGHV ciphertext $c=q \cdot p+r$, we first show in Lemma 1 how to obtain a "virtual" ciphertext of the form $c^{\prime}=2^{k} \cdot q^{\prime}+r^{\prime}$ with $\left[q^{\prime}\right]=[q]_{2}$, given the bits $s_{i}$ in the following subset-sum sharing of $2^{k} / p$ :

$$
\frac{2^{k}}{p}=\sum_{i=1}^{\Theta} s_{i} \cdot y_{i}+\varepsilon \quad \bmod 2^{k+1}
$$

where the $y_{i}$ 's have $\kappa$ bits of precision after the binary point, with $|\varepsilon| \leq 2^{-\kappa}$. This is done by first "expanding" the initial ciphertext $c$ using the $y_{i}$ 's, as in the "squashed decryption" procedure in [9], and then "collapsing" the expanded ciphertext into $c^{\prime}$, using the secret-key vector $s=\left(s_{i}\right)$. However we cannot reveal 
$s$ in clear, so instead we provide a DGHV encryption under $p^{\prime}$ of the secret-key bits $s_{i}$, as in the bootstrapped procedure. Then as showed in Lemma 2 the expanded ciphertext can be collapsed into a new ciphertext $c^{\prime \prime}$ under $p^{\prime}$ instead of $p$, for the same underlying plaintext; moreover as in the RLWE scheme the noise is reduced by a factor $\simeq p^{\prime} / p$.

Lemma 1. Let $p$ be an odd integer. Let $c=q \cdot p+r$ be a ciphertext. Let $k$ be an integer. Let $\kappa \in \mathbb{Z}$ be such that $|c|<2^{\kappa}$. Let $\boldsymbol{y}$ be a vector of $\Theta$ numbers with $\kappa$ bits of precision after the binary point, and let $s$ be a vector of $\Theta$ bits such that $2^{k} / p=\langle\boldsymbol{s}, \boldsymbol{y}\rangle+\varepsilon \bmod 2^{k+1}$, where $|\varepsilon| \leq 2^{-\kappa}$. Let $\boldsymbol{c}=\left(\left\lfloor c \cdot y_{i}\right\rceil \bmod 2^{k+1}\right)_{1 \leq i \leq \Theta}$. Let $c^{\prime}=\langle\boldsymbol{s}, \boldsymbol{c}\rangle$. Then $c^{\prime}=q^{\prime} \cdot 2^{k}+r^{\prime}$ with $\left[q^{\prime}\right]_{2}=[q]_{2}$ and $r^{\prime}=\left\lfloor r \cdot 2^{k} / p\right\rfloor+\delta$ where $\delta \in \mathbb{Z}$ with $|\delta| \leq \Theta / 2+2$.

Proof. We have:

$$
c^{\prime}=\sum_{i=1}^{\Theta} s_{i}\left\lfloor c \cdot y_{i}\right\rceil+\Delta \cdot 2^{k+1}=\sum_{i=1}^{\Theta} s_{i} \cdot c \cdot y_{i}+\delta_{1}+\Delta \cdot 2^{k+1}
$$

for some $\Delta \in \mathbb{Z}$ and $\left|\delta_{1}\right| \leq \Theta / 2$. Using $\langle\boldsymbol{s}, \boldsymbol{y}\rangle=2^{k} / p-\varepsilon-\mu \cdot 2^{k+1}$ for some $\mu \in \mathbb{Z}$ this gives:

$$
c^{\prime}-\delta_{1}-\Delta 2^{k+1}=c \cdot\left(\frac{2^{k}}{p}-\varepsilon-\mu \cdot 2^{k+1}\right)=q \cdot 2^{k}+r \cdot \frac{2^{k}}{p}-c \cdot \varepsilon-c \cdot \mu \cdot 2^{k+1}
$$

Therefore we can write:

$$
c^{\prime}=q^{\prime} \cdot 2^{k}+r^{\prime}
$$

where $\left[q^{\prime}\right]_{2}=[q]_{2}$ and $r^{\prime}=\left\lfloor r \cdot 2^{k} / p\right\rfloor+\delta$ for some $\delta \in \mathbb{Z}$ with $|\delta| \leq \Theta / 2+2$.

As in 4], given a vector $\boldsymbol{x} \in\left[0,2^{k+1}\left[{ }^{\Theta}\right.\right.$ we write $\boldsymbol{x}=\sum_{i=0}^{k} 2^{j} \cdot \boldsymbol{u}_{j}$ where all the elements in vectors $\boldsymbol{u}_{j}$ are bits, and we define $\operatorname{BitDecomp}(\boldsymbol{x}, k):=\left(\boldsymbol{u}_{0}, \ldots, \boldsymbol{u}_{k}\right)$. Similarly given a vector $\boldsymbol{z} \in \mathbb{R}^{\Theta}$ we define Powersof2 $(\boldsymbol{z}, k):=\left(\boldsymbol{z}, 2 \cdot \boldsymbol{z}, \ldots, 2^{k} \cdot \boldsymbol{z}\right)$. We have for any vectors $\boldsymbol{x}$ and $\boldsymbol{z}$ :

$$
\langle\operatorname{BitDecomp}(\boldsymbol{x}, k), \operatorname{Powersof} 2(\boldsymbol{z}, k)\rangle=\langle\boldsymbol{x}, \boldsymbol{z}\rangle
$$

The following lemma shows that given a ciphertext $c$ encrypted under $p$ and with noise $r$ we can compute a new ciphertext $c^{\prime \prime}$ under $p^{\prime}$ with noise $r^{\prime \prime} \simeq r \cdot p^{\prime} / p$, by using an encryption $\boldsymbol{\sigma}$ under $p^{\prime}$ of the secret-key $s$ corresponding to $p$.

Lemma 2. Let $p$ and $p^{\prime}$ be two odd integers. Let $k$ be an integer such that $p^{\prime}<2^{k}$. Let $c=q \cdot p+r$ be a ciphertext. Let $\kappa \in \mathbb{Z}$ be such that $|c|<2^{\kappa}$. Let $\boldsymbol{y}$ be a vector of $\Theta$ numbers with $\kappa$ bits of precision after the binary point, and let $\boldsymbol{s}$ be a vector of $\Theta$ bits such that $2^{k} / p=\langle\boldsymbol{s}, \boldsymbol{y}\rangle+\varepsilon \bmod 2^{k+1}$, where $|\varepsilon| \leq 2^{-\kappa}$. Let $\boldsymbol{\sigma}=p^{\prime} \cdot \boldsymbol{q}+\boldsymbol{r}+\left\lfloor\boldsymbol{s}^{\prime} \cdot p^{\prime} / 2^{k+1}\right\rceil$ be an encryption of the secret-key $\boldsymbol{s}^{\prime}=$ Powersof $2(\boldsymbol{s}, k)$, where $\boldsymbol{q} \leftarrow\left(\mathbb{Z} \cap\left[0,2^{\gamma} / p^{\prime}\right)\right)^{(k+1) \cdot \Theta}$ and $\boldsymbol{r} \leftarrow\left(\mathbb{Z} \cap\left(-2^{\rho}, 2^{\rho}\right)\right)^{(k+1) \cdot \Theta}$. Let $\boldsymbol{c}=\left(\left\lfloor c \cdot y_{i}\right\rceil \bmod 2^{k+1}\right)_{1 \leq i \leq \Theta}$ and let $\boldsymbol{c}^{\prime}=\operatorname{BitDecomp}(\boldsymbol{c}, k)$ be the expanded ciphertext. Let $c^{\prime \prime}=2\left\langle\boldsymbol{\sigma}, \boldsymbol{c}^{\prime}\right\rangle+[\bar{c}]_{2}$. Then $c^{\prime \prime}=q^{\prime \prime} \cdot p^{\prime}+r^{\prime \prime}$ where $r^{\prime \prime}=\left\lfloor r \cdot p^{\prime} / p\right\rfloor+\delta^{\prime}$ for some $\delta^{\prime} \in \mathbb{Z}$ with $\left|\delta^{\prime}\right| \leq 2^{\rho+2} \cdot \Theta \cdot(k+1)$, and $[r]_{2}=\left[r^{\prime \prime}\right]_{2}$. 
Proof. We have, from $\boldsymbol{\sigma}=p^{\prime} \cdot \boldsymbol{q}+\boldsymbol{r}+\left\lfloor\boldsymbol{s}^{\prime} \cdot p^{\prime} / 2^{k+1}\right\rceil$ :

$$
c^{\prime \prime}=2\left\langle\boldsymbol{\sigma}, \boldsymbol{c}^{\prime}\right\rangle+[c]_{2}=2 p^{\prime} \cdot\left\langle\boldsymbol{q}, \boldsymbol{c}^{\prime}\right\rangle+2\left\langle\boldsymbol{r}, \boldsymbol{c}^{\prime}\right\rangle+2\left\langle\left\lfloor\boldsymbol{s}^{\prime} \cdot \frac{p^{\prime}}{2^{k+1}}\right\rceil, \boldsymbol{c}^{\prime}\right\rangle+[c]_{2}
$$

Since the components of $\boldsymbol{c}^{\prime}$ are bits, we have using $2\lfloor x / 2\rceil=x+\nu$ with $|\nu| \leq 1$ :

$$
2\left\langle\left\lfloor\frac{p^{\prime}}{2^{k+1}} \cdot \boldsymbol{s}^{\prime}\right\rceil, \boldsymbol{c}^{\prime}\right\rangle=\left\langle\frac{p^{\prime}}{2^{k}} \cdot \boldsymbol{s}^{\prime}, \boldsymbol{c}^{\prime}\right\rangle+\nu_{2}=\frac{p^{\prime}}{2^{k}} \cdot\left\langle\boldsymbol{s}^{\prime}, \boldsymbol{c}^{\prime}\right\rangle+\nu_{2}
$$

where $\left|\nu_{2}\right| \leq \Theta \cdot(k+1)$. Using $\left\langle\boldsymbol{s}^{\prime}, \boldsymbol{c}^{\prime}\right\rangle=\langle\boldsymbol{s}, \boldsymbol{c}\rangle$ and since from Lemma1 1 we have $\langle\boldsymbol{s}, \boldsymbol{c}\rangle=q^{\prime} \cdot 2^{k}+r^{\prime}$ with $\left[q^{\prime}\right]_{2}=[q]_{2}$ and $r^{\prime}=\left\lfloor r \cdot 2^{k} / p\right\rfloor+\delta$ where $\delta \in \mathbb{Z}$ with $|\delta| \leq \Theta / 2+2$, we get:

$2\left\langle\left\lfloor\frac{p^{\prime}}{2^{k+1}} \cdot s^{\prime}\right\rceil, \boldsymbol{c}^{\prime}\right\rangle=\frac{p^{\prime}}{2^{k}} \cdot\left(q^{\prime} \cdot 2^{k}+r^{\prime}\right)+\nu_{2}=q^{\prime} \cdot p^{\prime}+\frac{p^{\prime}}{2^{k}} \cdot r^{\prime}+\nu_{2}=q^{\prime} \cdot p^{\prime}+r \cdot \frac{p^{\prime}}{p}+\nu_{3}$ where $\left|\nu_{3}\right| \leq\left|\nu_{2}\right|+\Theta / 2+3 \leq 2 \Theta \cdot(k+1)$. Therefore we obtain from equation (3):

$$
c^{\prime \prime}=2 p^{\prime} \cdot\left\langle\boldsymbol{q}, \boldsymbol{c}^{\prime}\right\rangle+2\left\langle\boldsymbol{r}, \boldsymbol{c}^{\prime}\right\rangle+q^{\prime} \cdot p^{\prime}+r \cdot \frac{p^{\prime}}{p}+\nu_{3}+[c]_{2}=q^{\prime \prime} \cdot p^{\prime}+r^{\prime \prime}
$$

where $q^{\prime \prime}:=q^{\prime}+2\left\langle\boldsymbol{q}, \boldsymbol{c}^{\prime}\right\rangle$ and $r^{\prime \prime}=\left\lfloor r \cdot p^{\prime} / p\right\rfloor+\delta^{\prime}$ for some $\delta^{\prime} \in \mathbb{Z}$ with:

$\left|\delta^{\prime}\right| \leq\left|2\left\langle\boldsymbol{r}, \boldsymbol{c}^{\prime}\right\rangle\right|+1+\left|\nu_{3}\right|+1 \leq 2^{\rho+1} \cdot \Theta \cdot(k+1)+2 \Theta \cdot(k+1)+2 \leq 2^{\rho+2} \cdot \Theta \cdot(k+1)$

Eventually from $\left[c^{\prime \prime}\right]_{2}=[c]_{2},[c]_{2}=[q]_{2} \oplus[r]_{2},\left[c^{\prime \prime}\right]_{2}=\left[q^{\prime \prime}\right]_{2} \oplus\left[r^{\prime \prime}\right]_{2}$ and $\left[q^{\prime \prime}\right]_{2}=$ $\left[q^{\prime}\right]_{2}=[q]_{2}$, we obtain $[r]_{2}=\left[r^{\prime \prime}\right]_{2}$ as required.

\subsection{The Modulus-Switching Algorithm for DGHV}

From Lemma 2 we can now specify the modulus-switching algorithm for DGHV.

SwitchKeyGen $\left(p k, s k, p k^{\prime}, s k^{\prime}\right)$ :

1. Take as input two DGHV secret-keys $p$ and $p^{\prime}$ of size $\eta$ and $\eta^{\prime}$. Let $\kappa=2 \gamma+\eta$ where $\gamma$ is the size of the public key integers $x_{i}$ under $p$.

2. Generate a vector $\boldsymbol{y}$ of $\Theta$ random numbers modulo $2^{\eta^{\prime}+1}$ with $\kappa$ bits of precision after the binary point, and a random vector $s$ of $\Theta$ bits such that $2^{\eta^{\prime}} / p=\langle\boldsymbol{s}, \boldsymbol{y}\rangle+\varepsilon \bmod 2^{\eta^{\prime}+1}$ where $|\varepsilon| \leq 2^{-\kappa}$. Generate the expanded secret-key $\boldsymbol{s}^{\prime}=$ Powersof2 $\left(s, \eta^{\prime}\right)$

3. Compute a vector encryption $\boldsymbol{\sigma}$ of $\boldsymbol{s}^{\prime}$ under $s k^{\prime}$, defined as follows:

$$
\boldsymbol{\sigma}=p^{\prime} \cdot \boldsymbol{q}+\boldsymbol{r}+\left\lfloor\boldsymbol{s}^{\prime} \cdot \frac{p^{\prime}}{2^{\eta^{\prime}+1}}\right\rceil
$$

where $\boldsymbol{q} \leftarrow\left(\mathbb{Z} \cap\left[0, q_{0}^{\prime}\right)\right)^{\left(\eta^{\prime}+1\right) \cdot \Theta}$ and $\boldsymbol{r} \leftarrow\left(\mathbb{Z} \cap\left(-2^{\rho^{\prime}}, 2^{\rho^{\prime}}\right)\right)^{\left(\eta^{\prime}+1\right) \cdot \Theta}$, where $q_{0}^{\prime}$ is from $x_{0}^{\prime}=q_{0}^{\prime} \cdot p^{\prime}+r^{\prime}$ in $p k^{\prime}$.

4. Output $\tau_{p k \rightarrow p k^{\prime}}=(\boldsymbol{y}, \boldsymbol{\sigma})$. 
$\operatorname{SwitchKey}\left(\tau_{p k \rightarrow p k^{\prime}}, c\right)$ :

1. Let $\boldsymbol{y}, \boldsymbol{\sigma} \leftarrow \tau_{p k \rightarrow p k^{\prime}}$

2. Compute the expanded ciphertext $c=\left(\left\lfloor c \cdot y_{i}\right\rceil \bmod 2^{\eta^{\prime}+1}\right)_{1 \leq i \leq \Theta}$ and let $\boldsymbol{c}^{\prime}=\operatorname{BitDecomp}\left(\boldsymbol{c}, \eta^{\prime}\right)$.

3. Output $c^{\prime \prime}=2\left\langle\boldsymbol{\sigma}, \boldsymbol{c}^{\prime}\right\rangle+[c]_{2}$.

\subsection{The DGHV Scheme without Bootstrapping}

We are now ready to describe our DGHV variant in the BGV framework, that is without bootstrapping. As in [4] we construct a leveled fully homomorphic scheme, i.e. an encryption scheme whose parameters depend polynomially on the depth of the circuits that the scheme can evaluate.

FHE. KeyGen $\left(1^{\lambda}, 1^{L}\right)$. Take as input the security parameter $\lambda$ and the number of levels $L$. Let $\mu$ be a parameter specified later. Generate a ladder of $L$ decreasing moduli of size $\eta_{i}=(i+1) \mu$ from $\eta_{L}=(L+1) \mu$ down to $\eta_{1}=2 \mu$. For each $\eta_{i}$ run DGHV.KeyGen $\left(1^{\lambda}\right)$ from Section 2 to generate a random odd integer $p_{i}$ of size $\eta_{i}$; we take the same parameter $\gamma$ for all $i$. Let $p k_{i}$ be the corresponding public key and $s k_{i}=p_{i}$ be the corresponding secret-key. For $j=L$ down to 2 run $\tau_{p k_{j} \rightarrow p k_{j-1}} \leftarrow$ SwitchKeyGen $\left(p k_{j}, s k_{j}, p k_{j-1}, s k_{j-1}\right)$. The full public key is $p k=\left(p k_{L}, \tau_{p k_{L} \rightarrow p k_{L-1}}, \ldots, \tau_{p k_{2} \rightarrow p k_{1}}\right)$ and the secret-key is $s k=\left(p_{1}, \ldots, p_{L}\right)$.

FHE. Encrypt $(p k, m \in\{0,1\})$. Run DGHV. Encrypt $\left(p k_{L}, m\right)$.

FHE. Decrypt $(s k, c)$. Suppose that the ciphertext is under modulus $p_{j}$. Output $m \leftarrow[c]_{p_{j}} \bmod 2$.

FHE. $\operatorname{Add}\left(p k, c_{1}, c_{2}\right)$. Suppose that the two ciphertexts $c_{1}$ and $c_{2}$ are encrypted under the same $p k_{j}$; if they are not, use FHE. Refresh below to make it so. First compute $c_{3} \leftarrow c_{1}+c_{2}$. Then output $c_{4} \leftarrow$ FHE.Refresh $\left(\tau_{p k_{j} \rightarrow p k_{j-1}}, c_{3}\right)$, unless both ciphertexts are encrypted under $p k_{1}$; in this case, simply output $c_{3}$.

FHE. Mult $\left(p k, c_{1}, c_{2}\right)$. Suppose that the two ciphertexts $c_{1}$ and $c_{2}$ are encrypted under the same $p k_{j}$; if they are not, use FHE. Refresh below to make it so. First compute $c_{3} \leftarrow c_{1} \cdot c_{2}$. Then output $c_{4} \leftarrow$ FHE.Refresh $\left(\tau_{p k_{j} \rightarrow p k_{j-1}}, c_{3}\right)$, unless both ciphertexts are encrypted under $p k_{1}$; in this case, simply output $c_{3}$.

FHE.Refresh $\left(\tau_{p k_{j+1} \rightarrow p k_{j}}, c\right)$. Output $c^{\prime} \leftarrow \operatorname{SwitchKey}\left(\tau_{p k_{j+1} \rightarrow p k_{j}}, c\right)$.

\subsection{Correctness and Security}

We show in the full version of this paper [6] how to fix the parameter $\mu$ so that the ciphertext noise for every modulus in the ladder remains roughly the same, and we prove that FHE is a correct leveled FHE scheme.

Theorem 3. For some $\mu=\mathcal{O}(\lambda+\log L)$, FHE is a correct L-leveled FHE scheme; specifically it correctly evaluates circuits of depth $L$ with Add and Mult gates over $G F(2)$. 
We show in the full version of this paper [6] that the resulting FHE is semantically secure under the following new assumption.

Definition 2 (Decisional Approximate GCD). The $(\rho, \eta, \gamma)$-Decisional Approximate GCD Problem is: For a random $\eta$-bit odd integer $p$, given polynomially many samples from $\mathcal{D}_{\gamma, \rho}(p)$, and given an integer $z=x+b \cdot\left\lfloor 2^{j} \cdot p / 2^{\eta+1}\right\rceil$ for $a$ given random integer $j \in[0, \eta]$, where $x \leftarrow \mathcal{D}_{\gamma, \rho}(p)$ and $b \leftarrow\{0,1\}$, find $b$.

The Decisional Approximate GCD assumption is defined in the usual way. It is clearly stronger than the standard Approximate GCD assumption. We were not able to base the security of the leveled DGHV scheme on the standard Approximate GCD assumption; this is due to equation (4) which requires a non-standard encryption of the secret-key bits.

Theorem 4. FHE is semantically secure under the Decisional Approximate GCD assumption and under the hardness of subset sum assumption.

\section{Improved Attack against the Approximate GCD Algorithm}

Recently, Chen and Nguyen 5] described an improved exponential algorithm for solving the approximate common divisor problem: they obtain a complexity of $\tilde{\mathcal{O}}\left(2^{\rho / 2}\right)$ for the partial version (with an exact multiple $x_{0}=q_{0} \cdot p$ ) and $\tilde{\mathcal{O}}\left(2^{3 \rho / 2}\right)$ for the general version (with near-multiples only) 1

In this section, we show that the latter complexity can be heuristically improved to $\tilde{\mathcal{O}}\left(2^{\rho}\right)$ provided that sufficiently many near-multiples are available, which is the case in the DGHV scheme. Our algorithm has memory complexity $\tilde{\mathcal{O}}\left(2^{\rho}\right)$, instead of only $\tilde{\mathcal{O}}\left(2^{\rho / 2}\right)$ for the Chen and Nguyen attack.

Indeed, assume that we have $s$ large near-multiples $x_{1}, \ldots, x_{s}$ of a given prime $p_{0}$, of the hidden form $x_{j}=p_{0} q_{j}+r_{j}$, where $q_{j} \in\left[0,2^{\gamma} / p_{0}\right.$ ) (for $\gamma$ polynomial in $\rho$ ) and $r_{j} \in\left[0,2^{\rho}\right)$ are chosen uniformly and independently at random. We claim that $p_{0}$ can then be recovered with overwhelming probability in time $\tilde{\mathcal{O}}\left(2^{\frac{s+1}{s-1} \rho}\right)$ (and with significant probability in time $\tilde{\mathcal{O}}\left(2^{\frac{s}{s-1} \rho}\right)$ ).

The algorithm is as follows. For $j=1, \ldots, s$, let:

$$
y_{j}=\prod_{i=0}^{2^{\rho}-1}\left(x_{j}-i\right)
$$

Clearly, $p_{0}$ divides the GCD $g=\operatorname{gcd}\left(y_{1}, \ldots, y_{s}\right)$. Each $y_{i}$ can be computed in time quasilinear in $2^{\rho}$ using a product tree, and the GCD can be evaluated as $\operatorname{gcd}\left(\cdots \operatorname{gcd}\left(\operatorname{gcd}\left(y_{1}, y_{2}\right), y_{3}\right), \ldots, y_{s}\right)$ using $s-1$ quasilinear GCD computations on numbers of size $\mathcal{O}\left(2^{\rho} \cdot \gamma\right)=\tilde{\mathcal{O}}\left(2^{\rho}\right)$. Hence, the whole computation of $g$ takes time $\tilde{\mathcal{O}}\left(s \cdot 2^{\rho}\right)$.

${ }^{1}$ Namely to solve the general version using the partial version algorithm it suffices to do exhaustive search on the $\rho$ bits of noise in $x_{0}=q_{0} \cdot p+r_{0}$. 
Now, we argue that with high probability on the choice of the $\left(q_{j}, r_{j}\right)$, all the prime factors of $g$ except $p_{0}$ are smaller than a bound $B$ that is not much larger than $2^{\rho}$. Then, $p_{0}$ can be recovered as $g / g^{\prime}$, where $g^{\prime}$ is the $B$-smooth part of $g$, which can in turn be computed in time quasilinear in $\max (B,|g|)$, e.g. using Bernstein's algorithm [1]. Overall, the full time complexity of the attack is thus $\tilde{\mathcal{O}}\left(\max \left(B, s \cdot 2^{\rho}\right)\right)$, or simply $\tilde{\mathcal{O}}(B)$ assuming that $s=O(\rho)$, and without loss of generality that $B>2^{\rho}$. All we need to find is how to choose $B$ to obtain a sufficient success probability.

The probability that all the prime factors of $g$ except $p_{0}$ are smaller than $B$ is the probability that, for every prime $p \geq B$ other than $p_{0}$, not all the $x_{j}$ 's are congruent to one of $0,1, \ldots, 2^{\rho}-1 \bmod p$. This happens with probability very close to $1-\left(2^{\rho} / p\right)^{s}$. Hence, the probability that all the prime factors of $g$ except $p_{0}$ are smaller than $B$ is essentially given by the following Euler product:

$$
P_{s, \rho}(B)=\prod_{\substack{p \geq B \\ p \neq p_{0}}}\left(1-\frac{2^{s \rho}}{p^{s}}\right)
$$

(which clearly converges to some positive value smaller than 1 since $s \geq 2$ and $B>2^{\rho}$ ). We prove in the full version of this paper [6] the following estimate on this Euler product.

Lemma 3. For any $B>2^{\rho+1 / s}$, we have:

$$
1-P_{s, \rho}(B)<\frac{2 s}{s-1} \cdot \frac{2^{s \rho}}{B^{s-1} \log B}
$$

In particular, if we pick $B=2^{\frac{s}{s-1} \rho}$, we obtain $P_{s, \rho}(B)>1-2 /(\rho \log 2)$ : thus, the problem can be solved in time $\tilde{\mathcal{O}}\left(2^{\frac{s}{s-1}} \rho\right)$ with significant success probability. And if we pick $B=2^{\frac{s+1}{s-1} \rho}$, we get $P_{s, \rho}(B)>1-2^{-\rho}$ : hence, the problem can be solved in time $\tilde{\mathcal{O}}\left(2^{\frac{s+1}{s-1} \rho}\right)$ with an overwhelming success probability.

We see in both cases that for any given $\varepsilon>0$, the complexity becomes $\mathcal{O}\left(2^{(1+\varepsilon) \rho}\right)$ if $s$ is large enough. Better yet, if $s=\omega(1)$ (for example $\Theta(\rho)$ ) nearmultiples are available, the problem can be solved in time $\tilde{\mathcal{O}}\left(2^{\rho}\right)$ with overwhelming probability.

As in [5] we can perform a time-memory trade-off. First split the product $y_{1}$ into $d$ sub-products $z_{k}$ 's, and guess which of these sub-products $z=z_{k}$ contains $p_{0}$. Let $g=\operatorname{gcd}\left(z, y_{2}, \ldots, y_{s}\right)$. The first $\operatorname{GCD}$ computation $\operatorname{gcd}\left(z, y_{2}\right)$ can be performed in time $\tilde{\mathcal{O}}\left(2^{\rho}\right)$ and memory $\tilde{\mathcal{O}}\left(2^{\rho} / d\right)$ by first computing $y_{2} \bmod z$ using a product tree; the remaining gcd's can be computed with the same complexity; the same holds for recovering the $B$-smooth part of $g$. Hence $p_{0}$ can be recovered in time $\tilde{\mathcal{O}}\left(d \cdot 2^{\rho}\right)$ and memory $\tilde{\mathcal{O}}\left(2^{\rho} / d\right)$.

\subsection{Experimental Results}

We have implemented the previous attack; see the full version of this paper [6] for the source code. Table 1 shows that our attack performs well in practice; it is roughly 200 times faster than the corresponding attack of Chen and Nguyen for the smallest set of parameters considered in [5]. 
Table 1. Running time of the attack, on a single core of an Amazon EC2 Cluster Compute Eight Extra Large Instance instance (featuring an Intel Xeon E5 processor at $2.5 \mathrm{GHz}$ and $60.5 \mathrm{~GB}$ of memory), with parameter $s=\rho$. For the third instance, the running time of the Chen-Nguyen attack [5] was estimated by multiplying the running time from [5] (1.6 $\mathrm{min})$ by $2^{\rho}$.

\begin{tabular}{|l|r|c|r|r||r|}
\hline Instance & $\rho$ & $\gamma$ & $\log _{2}$ mem. & running time & running time [5] \\
\hline \hline Micro & 12 & $10^{4}$ & 26.3 & $40 \mathrm{~s}$ & \\
\hline Toy (Section [8) & 13 & $61 \cdot 10^{3}$ & 29.9 & $13 \mathrm{~min} 22 \mathrm{~s}$ & \\
\hline Toy' ([5] without $x_{0}$ ) & 17 & $1.6 \cdot 10^{5}$ & 35.3 & $17 \mathrm{~h} 50 \mathrm{~min}$ & 3495 hours \\
\hline
\end{tabular}

\section{Implementation of DGHV with Compressed Public Key}

In this section we describe an implementation of the DGHV scheme with the compression technique of Section 3. we use the variant with $x_{0}=q_{0} \cdot p$. We refer to the full version of this paper [6] for a full description of the resulting scheme, and we provide the source code of our implementation in [17.

Asymptotic Key Size. To prevent lattice attacks against the sparse subsetsum problem, one must have $\Theta^{2}=\gamma \cdot \omega(\log \lambda)$; see 716 for more details. One can then take $\rho=\lambda, \eta=\tilde{\mathcal{O}}\left(\lambda^{2}\right), \gamma=\tilde{\mathcal{O}}\left(\lambda^{5}\right), \alpha=\tilde{\mathcal{O}}\left(\lambda^{2}\right), \tau=\tilde{\mathcal{O}}\left(\lambda^{3}\right)$ and $\Theta=\tilde{\mathcal{O}}\left(\lambda^{3}\right)$. Using our compression technique the public key size is roughly $2 \gamma+(\tau+\Theta) \cdot(\eta+\lambda)=\tilde{\mathcal{O}}\left(\lambda^{5}\right)$ bits.

Concrete Key Size and Execution Speed. We have updated the parameters from [7] to take into account the improved approximate-GCD attack from [5]; see Table 2], The attack from [5] is memory bounded; however we took a conservative approach and considered a memory unbounded adversary. As in [7] we take $n=4$ and $\theta=15$ for all security levels. We can see in Table 2 that compression reduces the public key size considerably. Table 3 shows no significant performance degradation with respect to [7].

Table 2. The concrete parameters of various test instances and their respective public key sizes, for DGHV with compressed public-key

\begin{tabular}{|l|c|c|c|c|c|c|c|r|}
\hline Instance & $\lambda$ & $\rho$ & $\eta$ & $\gamma \times 10^{-6}$ & $\alpha$ & $\tau$ & $\Theta$ & pk size \\
\hline \hline Toy & 42 & 27 & 1026 & 0.15 & 936 & 158 & 144 & $77 \mathrm{~KB}$ \\
\hline Small & 52 & 41 & 1558 & 0.83 & 1476 & 572 & 533 & $437 \mathrm{~KB}$ \\
\hline Medium & 62 & 56 & 2128 & 4.20 & 2016 & 2110 & 1972 & $2207 \mathrm{~KB}$ \\
\hline Large & 72 & 71 & 2698 & 19.35 & 2556 & 7659 & 7897 & $10.3 \mathrm{MB}$ \\
\hline
\end{tabular}


Table 3. Timings of our Sage 4.7.2 [15] code (single core of a desktop computer with an Intel Core2 Duo E8400 at $3 \mathrm{GHz}$ ), for DGHV with compressed public-key.

\begin{tabular}{|l|r|r|r|r|r|}
\hline Instance & KeyGen & Encrypt & Decrypt & Expand & Recrypt \\
\hline \hline Toy & $0.06 \mathrm{~s}$ & $0.05 \mathrm{~s}$ & $0.00 \mathrm{~s}$ & $0.01 \mathrm{~s}$ & $0.41 \mathrm{~s}$ \\
\hline Small & $1.3 \mathrm{~s}$ & $1.0 \mathrm{~s}$ & $0.00 \mathrm{~s}$ & $0.15 \mathrm{~s}$ & $4.5 \mathrm{~s}$ \\
\hline Medium & $28 \mathrm{~s}$ & $21 \mathrm{~s}$ & $0.01 \mathrm{~s}$ & $2.7 \mathrm{~s}$ & $51 \mathrm{~s}$ \\
\hline Large & $10 \mathrm{~min}$ & 7 min $15 \mathrm{~s}$ & $0.05 \mathrm{~s}$ & $51 \mathrm{~s}$ & $11 \mathrm{~min} 34 \mathrm{~s}$ \\
\hline
\end{tabular}

\section{Implementation of Leveled DGHV}

In this section we describe an implementation of the leveled DGHV scheme described in Section 5 in the BGV framework. We implement the modulusswitching procedure as described in Section [5.3. with an optimization of the ciphertext expansion procedure (see below). We also implement the bootstrapping operation; although not strictly necessary, this enables to get a FHE that can perform homomorphic evaluations indefinitely without needing to specify at setup time a bound on the multiplicative level.

\subsection{Faster Ciphertext Expansion}

We consider the modulus-switching procedure of Section 5.3 . The initial modulus $p$ has size $\eta$ and the new modulus $p^{\prime}$ has size $\eta^{\prime}<\eta$. The first modulus $p$ is shared among the $y_{i}$ elements as

$$
\frac{2^{\eta^{\prime}}}{p}=\sum_{i=1}^{\Theta} s_{i} \cdot y_{i}+\varepsilon \quad \bmod 2^{\eta^{\prime}+1}
$$

where the $s_{i}$ 's are bits, the $y_{i}$ 's have $\kappa$ bits of precision after the binary point, and $|\varepsilon| \leq 2^{-\kappa}$. In practice one can generate the $y_{i}$ 's pseudo-randomly (except $y_{1}$ ), as suggested in 7]. However the ciphertext expansion from Step 2 of SwitchKey algorithm (Section 5.3) is a time-consuming procedure.

Therefore instead of using pseudo-random $y_{i}$ 's we use the following (admittedly aggressive) optimization. Let $\delta$ be a parameter specified later. We generate a random $y$ with $\kappa+\delta \cdot \Theta \cdot \eta$ bits of precision after the binary point, and we define the $y_{i}$ 's for $2 \leq i \leq \Theta$ as:

$$
y_{i}=\left[y \cdot 2^{i \cdot \delta \cdot \eta}\right]_{2^{\eta^{\prime}+1}}
$$

keeping only $\kappa$ bits of precision after the binary point for each $y_{i}$ as previously. We fix $y_{1}$ so that equality (5) holds, assuming $s_{1}=1$. Then the ciphertext expansion from Step 2 of the SwitchKey algorithm (Section 5.3) can be computed as follows, for all $2 \leq i \leq \Theta$ :

$$
z_{i}=\left\lfloor c \cdot y_{i}\right\rceil \bmod 2^{\eta^{\prime}+1}=\left\lfloor c \cdot y \cdot 2^{i \cdot \delta \cdot \eta}\right\rceil \bmod 2^{\eta^{\prime}+1}
$$


Therefore computing all the $z_{i}$ 's (except $z_{1}$ ) is now essentially a single multiplication $c \cdot y$. In the full version of this paper [6] we describe a lattice attack against this optimization; we show that the attack is thwarted by selecting $\delta$ such that $\delta \cdot \Theta \cdot \eta \geq 3 \gamma$.

Finally we use the following straightforward optimization: instead of using BitDecomp and Powersof2 with bits, we use words of size $\omega$ bits instead. This decreases the running time of SwitchKey by a factor of about $\omega$, at the cost of increasing the resulting noise by roughly $\omega$ bits. We took $\omega=32$ in our implementation.

\subsection{Bootstrapping: The Decryption Circuit}

Recall that the decryption function in the DGHV scheme is:

$$
m \leftarrow\left\lfloor c-\left\lfloor\sum_{i=1}^{\Theta} s_{i} \cdot z_{i}\right\rceil\right]_{2}
$$

where $z_{i}=\left[c \cdot y_{i}\right]_{2}$ for $1 \leq i \leq \Theta$ is the expanded ciphertext, keeping only $n=\left\lceil\log _{2}(\theta+1)\right\rceil$ bits of precision after the binary point for each $z_{i}$. The $s_{i}$ 's form a sparse $\Theta$-dimensional vector of Hamming weight $\theta$, such that $1 / p=$ $\sum_{i=1}^{\Theta} s_{i} \cdot y_{i}+\varepsilon$ where the $y_{i}$ 's have $\kappa$ bits of precision after the binary point, and $|\varepsilon| \leq 2^{-\kappa}$. Note that for bootstrapping the decryption circuit is only used for the smallest modulus $p$ in the ladder. The following lemma shows that the message $m$ can be computed using a circuit of multiplicative depth exactly $n$.

Lemma 4. Let $a=\left[a_{0}, \ldots, a_{n}\right]$ and $b=\left[b_{0}, \ldots, b_{n}\right]$ be two integers of size $n+1$ bits, where every bit $a_{i}$ and $b_{i}$ has multiplicative depth at most $i$. Then every bit $c_{i}$ of the sum $c=(a+b) \bmod 2^{n+1}=\left[c_{0}, \ldots, c_{n}\right]$ has multiplicative depth at most $i$.

Proof. Let $\delta_{i}$ be the $i$-th carry bit, with $\delta_{0}=0$. We have $c_{i}=a_{i} \oplus b_{i} \oplus \delta_{i}$ for $0 \leq i \leq n$, where $\delta_{i}=a_{i-1} \cdot b_{i-1}+a_{i-1} \cdot \delta_{i-1}+b_{i-1} \cdot \delta_{i-1}$ for $1 \leq i \leq n$. Therefore by recursion $\delta_{i}$ has multiplicative depth at most $i$; this implies that $c_{i}$ has multiplicative depth at most $i$.

Therefore using a simple loop the sum of the $\Theta$ numbers $s_{i} \cdot z_{i}$ in equation (6) can be computed with a circuit of multiplicative depth $n$. Since a subsequent homomorphic operation (either addition or multiplication) must be possible between refreshed ciphertexts, the full bootstrapping procedure requires a leveled FHE scheme with multiplicative depth $L=n+1$. Note that for bootstrapping an encryption of the secret-key bits $s_{i}$ (corresponding to the last modulus $p_{1}$ in the ladder) must be provided under $p_{L}$, the first modulus in the ladder, so that the homomorphic evaluation of $m$ in equation (6) can start under the public key $p k_{L}$. 


\subsection{Implementation Results}

In this section we describe an implementation of the leveled DGHV scheme, including the bootstrapping operation. As mentioned previously we cannot use the variant with noise-free $x_{0}=q_{0} \cdot p$ since otherwise $p$ could be recovered using the ECM; namely the smallest modulus in the ladder has size only $2 \mu=164$ bits for the "Large" instance.

We summarize in Tables 4 and 5 the performance of our implementation of the leveled DGHV scheme. We denote by $\eta$ the size of the largest modulus in the ladder. The running time of the Recrypt operation is disappointing compared to the non-leveled implementation from Section 7 however we think that there is room for improvement.

Table 4. The concrete parameters of various test instances and their respective publickey sizes for leveled DGHV

\begin{tabular}{|l|c|c|c|c|c|r|r|}
\hline Instance & $\lambda$ & $\rho$ & $\eta$ & $\mu$ & $\gamma \times 10^{-6}$ & $\Theta$ & pk size \\
\hline \hline Toy & 42 & 14 & 336 & 56 & 0.061 & 195 & $354 \mathrm{~KB}$ \\
\hline Small & 52 & 20 & 390 & 65 & 0.27 & 735 & $1690 \mathrm{~KB}$ \\
\hline Medium & 62 & 26 & 438 & 73 & 1.02 & 2925 & $7.9 \mathrm{MB}$ \\
\hline Large & 72 & 34 & 492 & 82 & 2.20 & 5700 & $18 \mathrm{MB}$ \\
\hline
\end{tabular}

Table 5. Timings of our Sage 4.7.2 15] code (single core of a desktop computer with an Intel Core2 Duo E8400 at $3 \mathrm{GHz}$ )

\begin{tabular}{|l|r|r|r|r|r|}
\hline Instance & KeyGen & Encrypt & Decrypt & Mult \& Scale & Recrypt \\
\hline \hline Toy & $0.36 \mathrm{~s}$ & $0.01 \mathrm{~s}$ & $0.00 \mathrm{~s}$ & $0.04 \mathrm{~s}$ & $8.8 \mathrm{~s}$ \\
\hline Small & $5.4 \mathrm{~s}$ & $0.07 \mathrm{~s}$ & $0.00 \mathrm{~s}$ & $0.59 \mathrm{~s}$ & $101 \mathrm{~s}$ \\
\hline Medium & $1 \mathrm{~min} 12 \mathrm{~s}$ & $0.85 \mathrm{~s}$ & $0.00 \mathrm{~s}$ & $9.1 \mathrm{~s}$ & $32 \mathrm{~min} 38 \mathrm{~s}$ \\
\hline Large & $6 \mathrm{~min} 18 \mathrm{~s}$ & $3.4 \mathrm{~s}$ & $0.00 \mathrm{~s}$ & $41 \mathrm{~s}$ & $2 \mathrm{~h} 27 \mathrm{~min}$ \\
\hline
\end{tabular}

Acknowledgments. We would like to thank Tancrède Lepoint, Phong Nguyen and the EUROCRYPT referees for their helpful comments.

\section{References}

1. Bernstein, D.J.: How to Find Smooth Parts of Integers (2004), http://cr.yp.to/papers.html\#smoothparts

2. Brakerski, Z., Vaikuntanathan, V.: Efficient Fully Homomorphic Encryption from (Standard) LWE. In: Proceedings of FOCS 2011 (2011); Full version available at IACR eprint

3. Brakerski, Z., Vaikuntanathan, V.: Fully Homomorphic Encryption from Ring-LWE and Security for Key Dependent Messages. In: Rogaway, P. (ed.) CRYPTO 2011. LNCS, vol. 6841, pp. 505-524. Springer, Heidelberg (2011)

4. Brakerski, Z., Gentry, C., Vaikuntanathan, V.: Fully Homomorphic Encryption without Bootstrapping. Cryptology ePrint Archive, Report 2011/277 
5. Chen, Y., Nguyen, P.Q.: Faster Algorithms for Approximate Common Divisors: Breaking Fully-Homomorphic-Encryption Challenges over the Integers. Cryptology ePrint Archive, Report 2011/436

6. Coron, J.S., Naccache, D., Tibouchi, M.: Public-key Compression and Modulus Switching for Fully Homomorphic Encryption over the Integers. Full version of this paper. Cryptology ePrint Archive, Report 2011/440

7. Coron, J.-S., Mandal, A., Naccache, D., Tibouchi, M.: Fully Homomorphic Encryption over the Integers with Shorter Public Keys. In: Rogaway, P. (ed.) CRYPTO 2011. LNCS, vol. 6841, pp. 487-504. Springer, Heidelberg (2011); Full version available at IACR eprint

8. van Dijk, M., Gentry, C., Halevi, S., Vaikuntanathan, V.: Fully Homomorphic Encryption over the Integers. In: Gilbert, H. (ed.) EUROCRYPT 2010. LNCS, vol. 6110, pp. 24-43. Springer, Heidelberg (2010)

9. Gentry, C.: A fully homomorphic encryption scheme. Ph.D. thesis, Stanford University (2009), http://crypto.stanford.edu/craig

10. Gentry, C., Halevi, S.: Implementing Gentry's Fully-Homomorphic Encryption Scheme. In: Paterson, K.G. (ed.) EUROCRYPT 2011. LNCS, vol. 6632, pp. 129-148. Springer, Heidelberg (2011)

11. Lauter, K., Naehrig, M., Vaikuntanathan, V.: Can Homomorphic Encryption be Practical? Cryptology ePrint Archive, Report 2011/405

12. Lenstra, A.K.: Generating RSA Moduli with a Predetermined Portion. In: Ohta, K., Pei, D. (eds.) ASIACRYPT 1998. LNCS, vol. 1514, pp. 1-10. Springer, Heidelberg (1998)

13. Lenstra, H.W.: Factoring integers with elliptic curves. Annals of Mathematics 126(3), 649-673 (1987)

14. Smart, N.P., Vercauteren, F.: Fully Homomorphic Encryption with Relatively Small Key and Ciphertext Sizes. In: Nguyen, P.Q., Pointcheval, D. (eds.) PKC 2010. LNCS, vol. 6056, pp. 420-443. Springer, Heidelberg (2010)

15. Stein, W.A., et al.: Sage Mathematics Software (Version 4.7.2), The Sage Development Team (2011), http://www.sagemath.org

16. Stehlé, D., Steinfeld, R.: Faster Fully Homomorphic Encryption. In: Abe, M. (ed.) ASIACRYPT 2010. LNCS, vol. 6477, pp. 377-394. Springer, Heidelberg (2010)

17. https://github.com/coron/fhe 